Acta Crystallographica Section D

Biological

Crystallography

ISSN 0907-4449

Ted Baker ${ }^{\mathrm{a}}$ and

Zbigniew Dauter ${ }^{b}$

${ }^{\mathrm{a} S}$ School of Biological Sciences, University of Auckland, Private Bag 92-019, Auckland, New Zealand, and ${ }^{\mathbf{b}}$ National Cancer Institute, Brookhaven National Laboratory, Biology Department, Bldg 463, Upton, NY 11973, USA

\section{DNA and beyond}

This year the world celebrates the 50th anniversary of a momentous event in science, the unveiling of the structure of DNA. The implications have been profound and much has been written about its importance for science and society. But it is also interesting to think about the various strands that came together, metaphorically, in this work. There was already a belief, inspired by J. D. Bernal, Dorothy Crowfoot Hodgkin and others, that crystallography provided the key tool with which to address biological structure. For DNA, the theory of diffraction by helical structures had to be worked out. Experimental diffraction patterns, the work of Maurice Wilkins and Rosalind Franklin, were critical. And it required the imagination and insight of Crick and Watson to think about and recognise possible structures and their implications. These elements, theory, experimental crystallography, and insight from biological structure are the raison d'etre for this journal.

A famous picture from the Nobel Prize ceremony in 1962, published in Watson's book The Double Helix, provides a further striking reminder of this momentous era in biological crystallography. Together with Crick, Watson and Wilkins stand John Steinbeck, the novelist, and John Kendrew and Max Perutz, honoured for their development of protein crystallography in solving the structures of myoglobin and haemoglobin. It is also worth noting that Perutz and

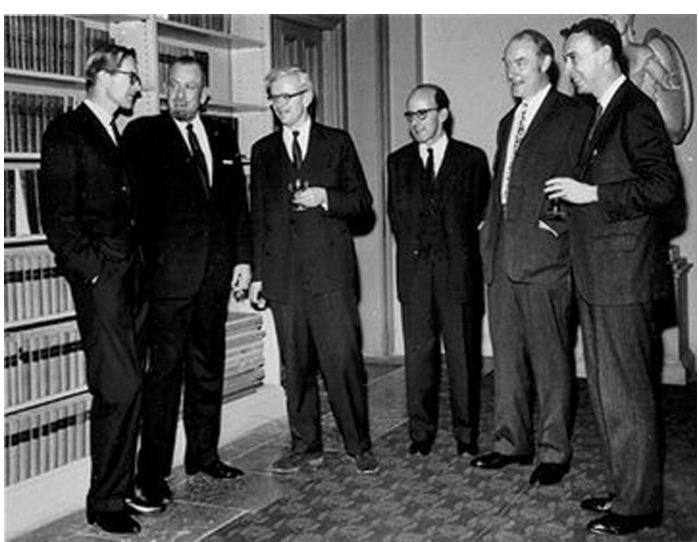

Maurice Wilkins, John Steinbeck, John Kendrew, Max Perutz, Francis Crick and Jim Watson shown after the Nobel Ceremony in Stockholm in December 1962. Source: MRC Laboratory of Molecular Biology, Cambridge, UK.
Kendrew were both chemists,

Crick and Wilkins physicists and Watson a biologist. This nicely illustrates the fact that in this field we use the methods of physics to address questions in biology and to answer them in the language of chemistry. For many of us, this is what makes biological crystallography so exciting, and while thinking about the importance and impact of the DNA structure, we can also be immensely proud of the contributions of the many people who have built up our field over the years. 